\title{
TRANSPLANTE DE CÉLULAS TRONCO-HEMATOPOIÉTICAS AUTÓLOGAS EM PACIENTES COM LINFOMA DE HODGKIN: EXPERIÊNCIA DA FACULDADE DE MEDICINA DO ABC - SP
}

\author{
Hematopoietic stem cell transplantation in Hodgkin's limphoma patients: \\ Experience at the ABC Medical School
}

\author{
Ronald Pallotta', Davimar Borducchi1, Vitor Mauad ', Renata Russo', Auro Del Giglio', Frederico Dulley²
}

\section{RESUMO}

Introdução: Apesar do excelente prognóstico do linfoma de Hodgkin, 20 a $25 \%$ dos pacientes são refratários primários e cerca de $30 \%$ deles recaem e requerem tratamento complementar. A estratégia de resgate inclui esquemas mais agressivos de quimioterapia, radioterapia e transplante de células tronco-hematopoiéticas. Objetivo: Descrever a experiência da Faculdade de Medicina do ABC (FMABC) no tratamento de pacientes com linfoma de Hodgkin que foram submetidos a transplante autólogo de medula óssea como parte do tratamento de resgate. Métodos: Estudo retrospectivo descritivo foi realizado a partir da revisão de prontuários de pacientes tratados na instituição no período de março de 2007 a dezembro de 2011. Nesse período, foram acompanhados 47 pacientes com diagnóstico de linfoma de Hodgkin na FMABC. Desses, 33 (70,2\%) estavam em remissão completa e 14 (29,8\%) não responderam ou recaíram. No grupo de 14 pacientes, 10 foram encaminhados para transplante autólogo de células troncohematopoiéticas em serviço especializado na FMUSP, uma vez que não existe serviço de transplante de medula óssea em nosso serviço. Resultados: A média de tempo entre a recaída e o transplante foi de 7,8 meses (variando de 4,9 a 14 meses). O regime de condicionamento para transplante foi BEAM (carmustina, citarabina, etoposide e melfalano) em $90 \%$ dos casos e a fonte de células foi o sangue periférico em $100 \%$ deles. A resposta foi obtida em $90 \%$ dos pacientes (oito RCC e um RP). As taxas foram: sobrevida global (SG) 88,8\%, sobrevida livre de doença (SLD) $55,6 \%$ e recaída $44,4 \%$, com média de seguimento de 24,05 meses (variando de oito a 42,7 ). Conclusão: A despeito da SG e SLD favoráveis, permanece o risco de recidiva e progressão da doença, obstáculos a serem ultrapassados com novas estratégias terapêuticas.

Keywords: Linfoma de Hodgkin, Recaída, Terapia de Salvação, Transplante de Medula Óssea

Instituições:

1. Divisão de Oncologia e Hematologia da Faculdade de Medicina do ABC (FMABC) - São Paulo/SP

2. Serviço de Transplante de Medula Óssea da Faculdade de Medicina da USP (FMUSP) - São Paulo/SP

\section{Correspondência:}

Ronald Pallotta

Rua João Rosa de Oliveira, no. 340, CEP 11442-350, Guarujá/SP

Tel.: (11) 95275-4573

E-mail:rp.cohs@yahoo.com.br

\section{INTRODUÇÃO}

Embora o linfoma de Hodgkin (LH) apresente excelentes taxas de resposta, 20 a $25 \%$ dos pacientes são refratários aos tratamentos iniciais, e cerca de $30 \%$ deles recaem e requerem tratamento complementar. ${ }^{1}$ Fatores prognósticos como estadio clínico, tempo de recaída, presença de sintomas $\mathrm{B}$, doença extra-nodal, performance status, anemia e gênero do paciente vêm sendo descritos, mas não são conclusivos para definição da estratégia terapêutica. ${ }^{2}$

O esquema de resgate inclui tratamentos mais agressivos de quimioterapia, radioterapia e transplante de células tronco-hematopoiéticas (TCTH) autólogo ou alogênico. 
O TCTH autólogo é o tratamento de escolha para pacientes com menos de 70 anos que recaem ou progridem após tratamento padrão. ${ }^{3,4}$

Com o objetivo geral de descrever a experiência da Divisão de Oncologia e Hematologia da Faculdade de Medicina do ABC (FMABC) foi realizado estudo baseado na revisão de prontuários médicos para determinar especificamente a sobrevida global (SG), sobrevida livre de doença (SLD) e taxa de recaída em pacientes com diagnóstico de linfoma de Hodgkin acompanhados no serviço e submetidos a TCTH autólogo no Serviço de Transplante de Medula Óssea da Faculdade de Medicina da USP.

\section{CASUÍSTICA E MÉTODOS}

No período de março de 2007 a dezembro de 2011, foram acompanhados na FMABC 47 pacientes com diagnóstico de linfoma de Hodgkin com idade média de 31,4 anos (variando de 18 a 64 anos), predomínio do sexo feminino de 1,35:1, e estadio clínico avançado em $74,5 \%$ dos casos. Desses, 33 (70,2\%) entraram em remissão e $14(29,8 \%)$ não responderam por completo ou recaíram, necessitando de tratamento complementar.

Do grupo de 14 pacientes, 10 foram encaminhados para TCTH autólogo em serviço referenciado, sendo o objeto deste estudo descritivo longitudinal.

A característica do grupo estudado demonstrou que $70 \%$ dos pacientes são do sexo masculino, a média de idade ao diagnóstico foi de 29,3 anos (variando de 19,6 a 53,2 anos), sendo que $100 \%$ apresentaram a forma clássica da doença, estando $80 \%$ em estadio avançado.
Com relação ao tratamento prévio ao transplante, $100 \%$ dos pacientes receberam ABVD (adriamicina, bleomicina, vincristina e dacarbazina) como esquema de tratamento inicial, sendo que, nos estadios precoces foram de quatro a seis ciclos associados à radioterapia em campo envolvido, e na forma avançada, de seis a oito ciclos. Após a recaída, $70 \%$ receberam ESHAP (etoposide, solumedrol, arabinosídeo-C, cisplatina), 10\% DHAP (araC, dexametasona e cisplatina), 10\% ICE (ifosfamida, carboplatina e etoposide) e 10\% TOPOSHAP (topotecano,solumedrol, arac, cisplatina) como esquemas terapêuticos de resgate (Tabela1).

Com relação à recaída, $60 \%$ tiveram-na tardia, $20 \%$ precoce e $20 \%$ não entraram em remissão.

Os dados coletados foram analisados pelo programa SPSS, sendo o estudo de SG e SLD determinados pelo método de Kaplan Meier.

\section{RESULTADOS}

A média de tempo entre a recaída e o transplante foi de 7,8 meses (variando de 4,9 a 14 meses). O regime de condicionamento realizado para transplante na FMUSP foi o esquema BEAM (carmustina, citarabina, etoposide e melfalano) em $90 \%$ dos casos e a fonte de células foi o sangue periférico em $100 \%$ deles.

Com relação à resposta, $90 \%$ responderam (oito $\mathrm{RC}$ e um $\mathrm{RP}$ ) e o paciente que progrediu foi a óbito em 41,7 meses pós-transplante, e com isso foi excluído das análises de SG e SLD. Dos nove pacientes que responderam, a SG foi de $88,8 \%$ e a SLD foi de $55,6 \%$, com taxa de recaída de $44,4 \%$, numa média de seguimento de 24,05 meses (variando de 8 a 42,7) - (Figura 1).

Tabela 1 - Características dos pacientes com diagnóstico de linfoma de Hodgkin acompanhados na Faculdade de Medicina do ABC, que foram submetidos a transplante de células tronco-hematopoiéticas autólogo

\begin{tabular}{ccccccc}
\hline Gênero & Idade & Estadiamento Clínico & Subtipo da Doença & Primeira Linha & Ciclos & Resgate \\
\hline H & 19 & IIIB & Esclerose Nodular & ABVD & 8 & ESHAP \\
M & 24 & IIIB & Esclerose Nodular & ABVD & 6 & ESHAP \\
H & 26 & IVB & Esclerose Nodular & ABVD & 8 & ESHAP \\
H & 25 & IIIBS & Esclerose Nodular & ABVD & 6 & ESHAP \\
M & 33 & IIIBS & Esclerose Nodular & ABVD & 6 & DHAP \\
H & 51 & IIA & Esclerose Nodular & ABVD & $4+$ RDT & ESHAP \\
M & 26 & IIIBS & Esclerose Nodular & ABVD & 7 & TOPO-SHAP \\
H & 23 & IIA & Esclerose Nodular & ABVD & $6+$ RDT & ESHAP \\
H & 23 & IVA & Esclerose Nodular & ABVD & 8 & ICE \\
H & 19 & IVB & Esclerose Nodular & ABVD & 8 & ESHAP \\
\hline
\end{tabular}

$A B V D=$ adriamicina, bleomicina, vincristina e dacarbazina; ESHAP = etoposide, solumedrol, arabinosídeo-C, cisplatina; DHAP = araC, dexametasona e cisplatina; TOPO-SHAP (topotecano, solumedrol, arac, cisplatina) 
Figura 1 - Sobrevida global e sobrevida livre de doença de pacientes com linfoma de Hodgkin, submetidos a transplante autólogo de células tronco-hematopoiéticas na Faculdade de Medicina do $A B C$

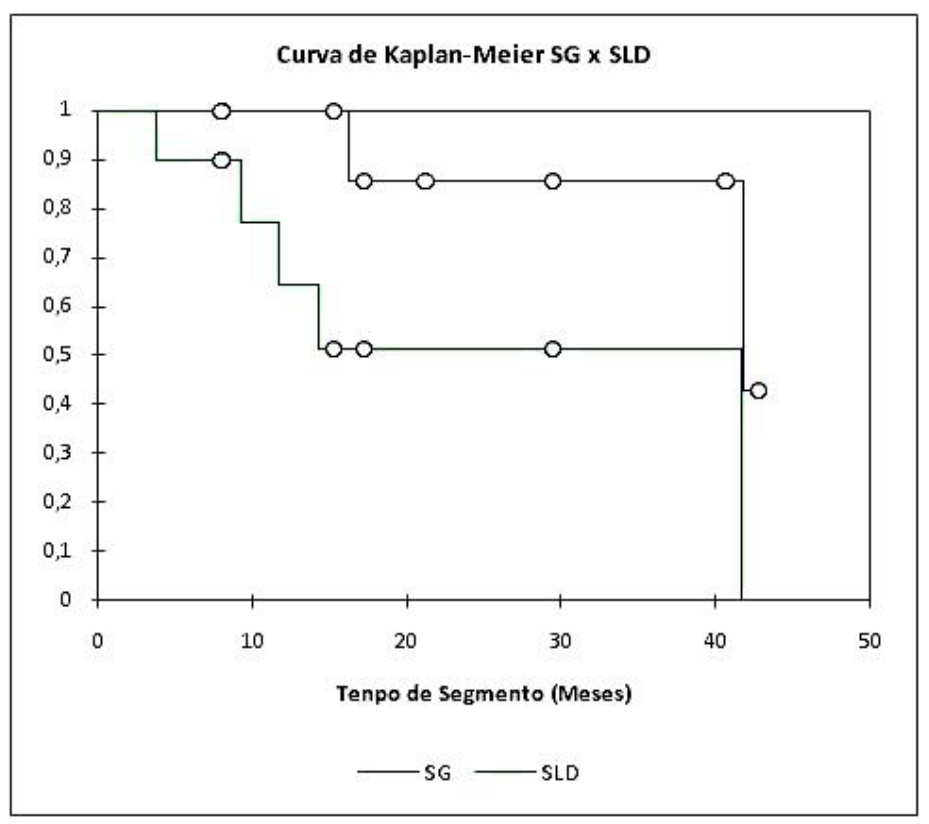

\section{DISCUSSÃO}

Aproximadamente $30 \%$ dos pacientes com $\mathrm{LH}$ recaem e $15 \%$ são refratários primários, no entanto, estes ainda apresentam chance de cura. Neste estudo, $29,8 \%$ dos doentes eram portadores desses perfis, dados similares à literatura. ${ }^{5}$

Os resultados de terapias de resgate são mais favoráveis para pacientes com remissão prolongada e para aqueles que recaem e respondem à terapia de salvamento convencional. Neste trabalho, correspondem a $60 \%$ dos pacientes encaminhados para TCTH. Nessa população, aproximadamente $50 \%$ serão curados com o autotransplante. ${ }^{6,7}$

Observou-se nos 10 pacientes refratários ou em recaída encaminhados para auto-TCTH uma taxa de resposta de $90 \%$, porém $44,4 \%$ deles recaíram, com SLD de $55,6 \%$, dados semelhantes aos encontrados na literatura mundial.

Entretanto, resultados de estudos randomizados tendem a limitar a indicação de TCTH para tratamento de LH, uma vez que pacientes com recidiva tardia $(>12$ meses após término do tratamento) podem obter a cura com terapias convencionais de resgate. Por outro lado, aparentemente, pacientes com doença progressiva ou com recidiva precoce (3-12 meses) devem ter TCTH considerado em sua estratégia de tratamento, sendo que o transplante autólogo é a primeira opção ( $40 \%$ da nossa população). ${ }^{8-11}$

Devido ao risco de recidiva ( $44,4 \%$ no nosso estudo) ou progressão da doença ( $10 \%$ no nosso estudo) após TCTH autólogo, o transplante de medula óssea alogênico, em especial com regime de condicionamento de intensidade reduzida (RIC) deve ser considerado como uma alternativa.

Os dados utilizando TCTH com regime de condicionamento em intensidade reduzida (RIC) são preliminares e permanecem muitas questões como: escolha do regime ideal, o papel do RIC como primeira opção de terapia de resgate e, a mais importante, a prova de existência de um efeito enxerto versus linfoma no LH..$^{12,13}$

Até a comprovação do real papel do TCTH alogênico na estratégia de tratamento do $\mathrm{LH}$, algumas condutas já vêm sendo tomadas. Sabidamente, a realização de tratamentos mais intensivos de resgate pré- TCTH autólogo tem melhorado os resultados. A importância da sensibilidade à quimioterapia antes do transplante é fator preditivo para o sucesso do tratamento. Pacientes que respondem à segunda linha de terapia apresentam resposta significativamente superior referente à SG, sobrevida livre de evento e sobrevida livre de progressão. ${ }^{14}$

Além disso, o desenvolvimento de esquemas combinando imunomoduladores associados ao tratamento quimioterápico ou de forma isolada, como o bortezomibe, lenalidomida, everolimus e panobinostat, têm sido estudados. ${ }^{15-17}$

Porém, a elaboração de imunoconjugados pode representar uma terapia mais eficaz, a ser utilizada como aliada no futuro. O conjugado brentuximab vedotin (BV) foi aprovado para tratamento de pacientes em recaída. Esse conjugado combina o anticorpo monoclonal antiCD30 com a anti-microtubulina monomethyl-auristatin $\mathrm{E}$, que tem demonstrado resultados expressivos com taxa de resposta de $75 \%$, onde $34 \%$ atingem resposta completa. $^{18}$

\section{CONCLUSÃO}

A despeito da SG e SLD favoráveis, permanece o risco de recidiva e progressão da doença, obstáculos a serem ultrapassados com novas estratégias terapêuticas. 


\section{ABSTRACT}

Background: Despite the excellent prognosis for the Hodgkin lymphoma, 20 to $25 \%$ patients are refractory and about $30 \%$ relapse, requiring further treatment. The rescuing therapy includes aggressive chemotherapy regimens, radiation therapy and hematopoietic stem cells transplantation. Purpose: To describe the experience at FMABC to treat patients with Hodgkin lymphoma who underwent autologous bone marrow transplantation as part of the rescue treatment. Methods: A retrospective descriptive study was performed to analyze the medical records of patients treated in the institution from March 2007 to December 2011.Forty-seven patients with Hodgkin lymphoma were diagnosed and treated at FMABC. From them, $33(70.2 \%)$ were in complete remission, but $14(29.8 \%)$ did not respond or relapsed. Among the 14 patients group, 10 were referred for autologous HSCT in a specialized service at USP, as there is no TMO in our service. Results: The mean time between the relapse and the transplantation was 7.8 months (ranging from 4.9 to 14 months). The transplant conditioning regimen was BEAM in $90 \%$ of cases and the stem cells source was the peripheral blood cells in $100 \%$. Ninety percent patients responded ( $8 \mathrm{CR}$ and $1 \mathrm{PR}$ ), with a $88.8 \%$ rate OS, $55.6 \%$ DFS, but $44.4 \%$ relapsed in a 24.05 month follow-up average period (ranging from 8 to 42.7 ). Conclusion: Despite the favorable OS and DFS, the risk of recurrence and progression of the disease remains an obstacle to be overcome with new therapeutic strategies.

Keywords: Hodgkin Lymphoma, Relapse, Salvage Therapy, Bone Marrow

\section{REFERÊNCIAS}

1. Kuruvilla, J, Keating, A and Crump, A. How I treat relapsed and refractory Hodgkin lymphoma. Blood.2011:137-46.

2. Eghbali H, Raemackers J, Carde P. EORTC lymphoma group, The EORTC strategy in the treatment of Hodgkin Lymphoma. Eur J Haematol Suppl.2005; 66:135-40.

3. Linch DC, Winfield D, Goldstone AH, Moir D, Hancock $B$, Mcmillan A et al. Dose intensification with autologous bone marrow transplantation in relapsed and resistant Hodgkin's diease, results of a BNLI randomized trial. Lancet 1993;341(8852):1051-4.

4. Schimitz N, Pfistner B, Sextro M, Sieber M, Carella AM, Haenel $M$ et al. Aggressive conventional chemotherapy compared with high dose chemotherapy with autologous bone marrow transplantation for relapsed chemosensitive Hodgkin's disease: a randomized trial. Lancet. 2002;359(9323):2065-71.

5. Strarbnik AP, Pro B. Heads or tails? Choosing a salvage therapy for relapsed/refractory Hodgkin lymphoma. Exp. Hematol. 2013;6(1):1-3

6. Brusamolino E, Bacigalupo A, Barosi G, Biti G, Gobbi P, Levis A et al. Classical Hodgkin's Lymphoma in adults: guidelines of the Italian Society of Hematology, the Italian Society Experimental Hematology and the Italian Group for Bone Marrow Transplantation on initial work-up, management, and follow up. Hematologica; 2009;94(4):550-65.

7. Canellos GP, Niedzwiecki D. Long-term follow up of Hodgkin's disease trial. N Engl J Med. 2002; 346(18):1417-8.

8. Bittencourt RL, Fogliato L, Paz A, Souza MP,Lener D. Transplante de células-tronco hematopoiéticas em linfoma de Hodgkin. Rev.Bras. Hematol. Hemoter. 2010;32 (supl.1):97105.

9. Nachbaur D, Oberaigner W, Fritsch E, Nussbaumer W, Gastl G. Allogeneic or autologous stem cell transplantation (SCT) for relapsed and refractory Hodgkin's disease and non-Hodgkin's lymphoma: a single-centre experience. Eur J Haematol. 2001;66(1):43-9.
10.Claviez A, Sureda A, Schmitz N. Haematopoietic SCT for children and adolescents with relapsed and refractory Hodgkin's lymphoma. Bone Marrow Transplant. 2008; 42 (2):S16-24.

11.Nachbaur D, Greinix HT, Koller E, Krieger O, Linkesch $\mathrm{W}$, Kasparu $\mathrm{H}$ et al Long-term results of autologous stem cell transplantation for Hodgkin's disease (HD) and low-l intermediate-grade B non-Hodgkin's lymphoma (NHL): a report from the Austrian Stem Cell Transplantation Registry (ASCTR).Byern B, Gokerman JP. Salvage therapy in Hodgkin's lymphoma. The oncologist. 2007;12(2):156-67.

12.Brusamolino $\mathrm{E}$, CarellaM. Treatment of refractory and relapsed Hodgkin's Lymphoma: facts and perspactives. Haematologica 2007;92(1):6-10.

13.Moskowitz $\mathrm{CH}$, Kewalramani T, Nimer SD, Gonzalez $\mathrm{M}$, Zelenetz AD, Yahalom J. Effectiveness of high dose chemoradiotherapy and autologous stem cell transplantation for patients with biopsy-proven primary refractory Hodgkin's disease. Br. J. Haematol.2004;124(5):645-52.

14.Fanale M, Fayad L, Pro B et al. Phase I study of bortezomib plus ICE (BICE) for the treatment of relapsed/refractory Hodgkin lymphoma. Br. J. Haematol. 2011;154(2):284-6.

15.Younes A, Sureda A, Ben-Yehuda D et al. Panobinostat in patients with relapsed/refractory Hodgkin's lymphoma after autologous stem-cell transplantation: results of a Phase II study. J. Clin. Oncol.2012;30(18):2197-203.

16. Johnston PB, Pinter-Brown L, Rogerio J. Open-label, singlearm, Phase II study of everolimus in patients with relapsed/ refractory classical hodgkin lymphoma. ASH Annual Meeting Abstracts, 2011; Abstract 2717:118.

17.Fehniger TA, Larson S, Trinkaus $\mathrm{K}$ et al. A Phase 2 multicenter study of lenalidomide in relapsed or refractory classical Hodgkin lymphoma. Blood. 2011;118(19):5119-25.

18.Younes A, Sureda A, Ben-Yehuda D et al. Panobinostat in patients with relapsed/refractory Hodgkin's lymphoma after autologous stem-cell transplantation: results of a Phase II study. J. Clin. Oncol. 2012;30(18):2197-203. 